\title{
Conduites addictives en milieu professionnel : aspects réglementaires
}

\section{Addiction at the workplace : legal aspects}

Paul FRIMAT*, Catherine NISSE

Service de Pathologie Professionnelle et Environnement, CHRU, Université de Lille 2, 1, avenue Oscar Lambret - 59037 LILLE Cedex

* Auteur à qui adresser la correspondance : Paul FRIMAT, Service de Pathologie Professionnelle et Environnement, CHRU, Université de Lille 2, 1, avenue Oscar Lambret - 59037 LILLE Cedex Tél : 0320445602 - Fax : 0320445591 - e-mail : labmedtrav@wanadoo.fr

(Reçu le $1^{\text {er }}$ février 2002 ; accepté le 20 février 2002)

\section{RÉSUMÉ}

Est-il raisonnable de confier des vies humaines à un sujet en état d'ivresse ou sous l'emprise de stupéfiants ou de médicaments agissant sur le système nerveux ?

L'entreprise est directement concernée par les conduites addictives. Le médecin du travail doit agir dans le cadre de l'hygiène et de la sécurité en s'appuyant sur des bases juridiques tout en respectant la déontologie. Cette dernière est claire, l'aspect juridique l'est moins mais certainement suffisant pour agir. La responsabilité du médecin est engagée. Il doit tout mettre en cuvre pour prévenir et faire cesser immédiatement une situation faisant encourir un danger particulier au salarié, à ses collègues de travail, à la collectivité et à l'environnement. Les dispositions légales sont les suivantes: Le Code Pénal (loi sur les stupéfiants du 31/13/1970), le Code de la Santé Publique (Art. L. 623 à L. 630), le Code de Déontologie ( secret professionnel), le Code du Travail : si le dépistage des substances toxicomanogènes n'est pas précisé, le médecin dispose de nombreux moyens pour répondre: que ce soit dans son rôle de conseiller (article R. 241-41) ou dans la procédure d'aptitude (article R. 241-48 à 51). Le médecin du travail peut agir face au problème de la toxico-

\section{SUMMARY}

Is it reasonable to confide human lives to people in drunken state or under drug or medicine ascendancy? Today, the workplace is directly affected by drug addiction. The occupational physician has to act in the field of hygiene and security, using the juridical basis and according to the deontology. His responsibility is engaged. He must implement everything, in order to prevent or to make cease immediately a situation dangerous for a worker, his colleagues, the collectivity and the environment.

Legal disposition are numerous and French laws approaching the question of addiction are the following : the Penal code (law on drugs, 31/13/1970); the Public Health Code (Art L. 623 to L.630) ; the Deontology Code (professional secret); the Occupational Code : even if the tracking of drug is not well specified, the physician can act face to the problem of drug addiction (circulaire $n^{\circ} 90 / 13$ of 09/07/1990), especially by playing his part of adviser (Art R 241-41) and in the procedure of aptitude (Art. $R$ 241-48 to 51) ; the Transportation Code (Arrêté of 07/05/97 and décret of 270/080/01 and 05/09/01 and law of 15/11/01).

They are rights and limits : the drug addiction increases the 
manie (circulaire $n^{\circ} 90 / 13$ du 9 juillet 1990), le Code des Transports (délivrance du permis de conduire) : L'arrêté du 7/05/97 (JO du 29/05/97) fixe la liste des incapacités physiques incompatibles avec l'obtention ou le maintien du permis de conduire. Depuis le décret du 27 août 2001 et l'arrêté du 5 septembre 2001, la recherche de stupéfiants doit être pratiquée sur les conducteurs impliqués dans un accident mortel. L'article 21 de la loi du 15 novembre 2001 relative à la sécurité quotidienne souligne le fait que les officiers de police judiciaire peuvent également faire procéder à cette recherche en cas d'accident corporel.

En pratique, il existe des droits et limites. La toxicomanie multiplie le risque de troubles du comportement, d'absentéisme, d'accident, de baisse de la vigilance. Dans le cadre des missions du médecin du travail, le dépistage éventuel des «dépendances» en entreprise ne doit pas conduire à une sélection, mais doit se placer dans une démarche de prévention. Le règlement intérieur doit rappeler l'interdiction de détention et d'usage de substances illicites au sein de l'entreprise et l'obligation de respecter les examens exigés par le médecin du travail, notamment pour les postes à risque. Il est indispensable d' adopter au sein de l'entreprise une attitude cohérente et une très large concertation avec la direction, les délégués du personnel et les CHS-CT. Il faut multiplier les campagnes d'information et créer des cellules de prévention.

Le médecin traitant est régulièrement confronté à la toxicomanie, il se doit de la prendre en charge. Le médecin du travail a le devoir de s'intéresser à ce phénomène dont les conséquences sont multiples pour la santé et la sécurité de l'individu, de l'entreprise et de la collectivité.

\section{MOTS-CLÉS}

Toxicomanie, réglementation, milieu professionnel.

\section{Introduction}

Les drogues font partie intégrante de notre société moderne, qu'il s'agisse de drogues bénéficiant d'une tolérance générale, comme l'alcool, ou de drogues illégales. Le lieu de travail n'échappe pas à la règle. Le médecin du travail, en entreprise, est confronté dès à présent à ce problème. En raison des risques importants pour le salarié dépendant, pour l'entreprise, pour la collectivité, il se trouve face à un dilemme difficile : concilier l'intérêt de l'individu et l'intérêt de l'entreprise.

Aucune notion précise concernant les postes de sécurité n'existe dans le code du travail, mais le médecin du travail doit les surveiller. Toutefois, la législation de 1991 impose déjà le dépistage des toxicomanies pour les contrôleurs aériens. Depuis 1992, un dépistage est obligatoire pour tous les équipages des transports aériens dans le monde. Les États-Unis exigent l'état de «drug-free» pour tous les personnels approchant leurs aéronefs.

Le Livre Blanc de la sécurité routière envisageait plus que sérieusement d'étendre le dépistage des pharmaco- risk of behaviour disorders, of absenteeism, of accidents, of decline of the vigilance.In the framework of the missions of the occupational physician, the possible tracking of "dependence", in companies, must not drive to a selection but has to be placed in a step of prevention. The "inner rules" have to remind the prohibition of detention and usage of illicit substances in the company, and the obligation to comply with the examinations ordained by the occupational physician, particularly for risk positions. It is indispensable to adopt within the companies a coherent behaviour and a very large dialogue with the direction, the staff delegates and the safety and hygiene comity. It is necessary to multiply campaigns of information and to create cells of prevention.

The general practitioner is regularly confronted to drug addiction and must take over the medical part of it. The occupational physician must show interest in this phenomenon, whose consequences are multiple for the health and the safety of the person, the workplace and the community.

\author{
KEY-WORDS \\ Drug addiction, workplace, law.
}

dépendances en cas d'accident grave ou de comportement anormal. Ceci a été confirmé par de récentes dispositions réglementaires.

\section{Recherche des conduites toxicophiles en entreprise Notions générales}

Est-il raisonnable de confier des vies humaines à un sujet en état d'ivresse ou sous l'empire de stupéfiants ou de médicaments agissant sur le système nerveux ? L'entreprise est directement concernée par les toxicomanies (stupéfiants et enivrants). Le médecin du travail doit agir dans le cadre de l'hygiène et de la sécurité en s'appuyant sur des bases juridiques tout en respectant la déontologie. Cette dernière est claire, l'aspect juridique l'est moins mais certainement suffisant pour agir.

La responsabilité du médecin est engagée. Il doit tout mettre en œuvre pour prévenir et faire cesser immédiatement une situation faisant courir un danger particulier au salarié, à ses collègues de travail, à la collectivité et 
à l'environnement. Connaissant l'aspect législatif (Code de la Santé Publique, Code Pénal, Code du Travail), et l'aspect déontologique (Code de Déontologie), le médecin du travail sera prêt à évaluer, s'il l'estime nécessaire, le risque toxicomaniaque au sein de l'entreprise et en particulier celui aux «postes de sécurité et de sûreté» qui exigent une vigilance intégrale. L'appréciation de l'aptitude et le dépistage des toxiques se feront suivant des règles très précises : le toxique est un danger pour le salarié et pour autrui. Le salarié sera avisé du dépistage. Le médecin, astreint au secret professionnel absolu, ne fera pas connaître le résultat de l'analyse à l'employeur sinon en terme d'aptitude ou d'inaptitude au poste. Par ailleurs, c'est à l'employeur de fixer la réglementation en matière d'hygiène et de sécurité dont il est responsable vis-àvis de la loi, par un règlement intérieur.

L'introduction de drogue illicite dans l'entreprise est une faute lourde. Le règlement intérieur doit rappeler l'interdiction et l'usage de substances illicites au sein de l'entreprise et l'obligation de respecter les examens exigés par le médecin du travail notamment pour les «postes de sécurité et de sûreté».

C'est en multipliant l'information, la prévention et la concertation que l'on pourra réduire le phénomène toxicomanie dont les conséquences sont multiples pour la santé et la sécurité de l'individu, de l'entreprise et de la collectivité.

Si l'employeur ne peut décider seul de la désignation des emplois de sécurité ou de sûreté, il faut rappeler que le médecin du travail reste son conseiller dans l'évaluation des risques. En cas d'accident, leur responsabilité pourrait être engagée.

\section{Les dispositions légales}

\section{- Le Code Pénal}

La loi sur les stupéfiants (31/12/1970) stipule que toutes les substances toxicomanogènes sont illicites. Il ne peut y avoir d'autres consommations que celles à visée thérapeutique.

Elle distingue :

- les psycholeptiques : opiacés et dérivés

- les psychoanaleptiques : cocaïne et dérivés

- les psychodysleptiques (hallucinogènes) : cannabis, ecstasy, LSD, PCP...

\section{- Le Code de la Santé Publique}

Les textes relatifs à l'usage et au trafic des stupéfiants sont réunis dans le Code de

la Santé Publique (Art. L 623 à L. 630).

\section{- Le Code de Déontologie}

Le fait d'être lié par contrat avec un employeur ou une société morale n'enlève rien au secret professionnel et à l'indépendance de décision. L'article 75 précise que le médecin doit agir en priorité dans l'intérêt de la santé des personnes qu'il examine, dans l'intérêt de leur sécurité au sein de l'entreprise ou des collectivités dont il est responsable.

\section{- Le Code du Travail}

Si le dépistage des substances toxicomanogènes n'est pas précisé, le médecin dispose de nombreux moyens pour répondre aux situations particulières.Que ce soit dans son rôle de conseiller (Article R. 241-41), dans le cadre des examens complémentaires (Article R. 24152), dans la procédure d'aptitude à l'embauche ou pendant la vie professionnelle du salarié (Article R. 241-48 à 51), le médecin du travail peut agir face au problème de la toxicomanie.

\section{- Le Code des Transports (délivrance du permis de conduire)}

L'arrêté du 7/05/97 (JO du 29/05/97) fixe la liste des incapacités physiques incompatibles avec l'obtention ou le maintien du permis de conduire ainsi que des affections susceptibles de donner lieu à la délivrance du permis de conduire de durée de validité limitée. Il existe ainsi une relative incompatibilité à la conduite (véhicules légers et/ou lourds) en cas de consommation régulière de substances psychotropes. En cas de dépendance ou d'abus, l'incompatibilité devient totale.

\section{- Le Code de la Route}

Le décret du 27/8/2001 (J.O. du 28/8/2001) précise les modalités de recherche de stupéfiants pratiquée sur les conducteurs impliqués dans un accident mortel de la circulation routière.

L'arrêté du 5/9/2001 (J.O. du 18/9/2001) fixe les modalités de dépistage des stupéfiants et des analyses et examens prévus par le décret précédent. Les seuils minima de détection sont ainsi fixés :

$\begin{array}{lcc} & \text { Urine } & \text { Sang } \\ \text { - } \Delta 9 \text { tétrahydrocannabinoe } & 50 \mathrm{ng} / \mathrm{ml} & 1 \mathrm{ng} / \mathrm{ml} \\ \text { - Amphétamines } & 1000 \mathrm{ng} / \mathrm{ml} & 50 \mathrm{ng} / \mathrm{ml} \\ \text { - Cocaïne } & 300 \mathrm{ng} / \mathrm{ml} & 50 \mathrm{ng} / \mathrm{ml} \\ \text { - Opiacés } & 300 \mathrm{ng} / \mathrm{ml} & 20 \mathrm{ng} / \mathrm{ml}\end{array}$

Enfin, la loi du 15/11/2001 (J.O. du 16/11/2001) relative à la sécurité routière précise en son article 21 la modification de l'article L. 235-1 du Code de la Route. Ainsi, les officiers de police judiciaire pourront faire 
procéder à des épreuves de dépistage et des analyses ou examens médicaux sur tout conducteur d'un véhicule impliqué dans un accident corporel de la circulation, en vue d'établir s'il conduisait sous l'influence de substances ou plantes classées comme stupéfiant.

En conséquence, les épreuves de dépistage seront obligatoires en cas de décès, mais elles ne seront que facultatives en cas d'accident.

\section{En pratique : droits et limites}

En entreprise, le médecin du travail doit être prêt à évaluer, s'il l'estime nécessaire, le risque toxicomaniaque, mais aussi à déterminer l'aptitude du candidat ou du salarié à son poste de travail. Comme nous le savons, la toxicomanie multiplie le risque de troubles du comportement, d'absentéisme, d'accident, de baisse de la vigilance... Le médecin du travail doit donc toujours penser aux conséquences pour l'entreprise, les collègues de travail et l'environnement.

\section{Démarche d'aptitude}

Mission essentielle du médecin du travail, l'aptitude sera appréciée lors de l'ensemble des visites médicales. Le principe du dépistage de certains toxiques peut être décidé pour les postes à risques. Il est impératif de le faire de façon cohérente en respectant la loi, la déontologie, en informant le salarié, en respectant le secret professionnel.

Ces problèmes ont déjà été examinés par le Conseil Supérieur de la Prévention des Risques Professionnels. Une circulaire du 9/7/1990 spécifie la position du Ministère du Travail vis-à-vis du dépistage de la toxicomanie.

Toutefois, nous rappellerons que pour l'aviation civile et militaire, un arrêté du 17/7/1991 (J.O. du 31/7/1991) précise les conditions médicales particulières pour les contrôleurs aériens. Cet arrêté précise que le dosage du toxique doit être systématique pour tous les candidats à l'école et lors de la première affectation. Durant la vie professionnelle, ce dosage est soumis à l'appréciation du médecin du travail.

De même, pour ce qui concerne la conduite de véhicule, le médecin devra apprécier l'aptitude du salarié en tenant compte de l'état de santé, de la complexité du poste de travail. L'avis du spécialiste sera souvent utile. Le prescripteur de substances psychotropes se devra d'informer le salarié des conséquences médico-légales et engage ainsi sa propre responsabilité.

Par ailleurs, l'aptitude d'un salarié sous traitement substitutif nécessitera une démarche précise, une collaboration avec l'équipe médicale de soutien et entraînera souvent une décision difficile mais utile pour le reclassement de ce salarié en difficulté.

\section{Poste de sécurité/sûreté}

Comme pour l'alcool, la toxicomanie augmente le risque de certains postes... On parle ainsi de «poste de sécurité ou de sûreté».

Aucune définition légale n'existe, même si le conseil d'état s'appuie sur cette notion pour réglementer le dépistage d'alcoolémie en entreprise (CE, 1/2/1980, AJDA 1980-439).

Dans tous ces cas, le médecin du travail devra donc évaluer les risques professionnels et définir avec l'entreprise les postes concernés. L'employeur, quant à lui, décidera d'inscrire au règlement intérieur la justification du dépistage éventuel.

\section{Le dépistage des toxicomanies en entreprise}

La circulaire $\mathrm{n}^{\circ}$ 90/13 du 9 juillet 1990 relative au dépistage des toxicomanies en entreprise, précise tout d'abord que «rien ne justifie un dépistage de la toxicomanie organisé de manière systématique dans l'entreprise». Toutefois, dans certains cas particuliers (postes à risque) un dépistage peut être justifié. Mais la circulaire n'a pas établi de liste d'activités ou postes de travail entraînant ce dépistage.

Si le dépistage doit être fait, il est nécessaire d、 réciser les principes qui doivent guider le médecin du travail et la garantie qui doit entourer celui-ci.

Ainsi, les règles fondamentales de mise en place de ce dépistage pourraient être les suivantes :

- l'usage de produits toxiques s'avère un danger pour autrui (poste à risque)

- le travailleur doit en être avisé et s'il n'accepte pas, le médecin ne pourra délivrer d'aptitude

- l'employeur ne doit pas connaître le résultat

- le médecin du travail est astreint au secret professionnel absolu

- le médecin du travail, médecin à part entière, est le seul juge, au terme d'un examen clinique et d'un interrogatoire, des examens à proposer au salarié pour l'appréciation de son état de santé : il pourra demander les examens nécessaires au dépistage d'affections de nature à créer un danger pour le salarié ou pour autrui et ce, en lien direct et nécessaire avec l'emploi

- la fiche d'aptitude, établie par le médecin du travail à l'issue de chaque examen médical, ne doit mentionner que l'aptitude ou l'inaptitude partielle ou totale du candidat ou du salarié. Elle ne doit comporter aucun renseignement confidentiel tel que le diagnostic, la nature ou l'origine de l'inaptitude. Elle ne saurait donc révéler une toxicomanie comme telle. 
L'employeur, quant à lui, doit préciser dans le cadre de son règlement intérieur et avec les partenaires sociaux, l'interdiction de consommer de la drogue au sein de l'entreprise et l'obligation de se soumettre aux examens demandés par le médecin du travail pour les postes à risque (il y aura lieu de les définir).

Si tous ces principes sont respectés dans l'intérêt du salarié, il ne faut pas oublier que les résultats devront lui être communiqués. Toutefois, nous rappelons que le dépistage urinaire n'est pas un moyen infaillible de repérer les toxicomanies et qu'un dépistage reste ponctuel. Il y aura nécessité de recontrôler en cas de positivité le prélèvement dans un laboratoire indépendant et spécialisé.

\section{Conclusion}

Dans le cadre des missions du médecin du travail, le dépistage éventuel des «dépendances» en entreprise ne doit pas conduire à une sélection, mais doit se placer dans une démarche de prévention. On peut aussi, dans un souci de transparence, en respectant les règles déontologiques et la rigueur scientifique, préciser quelques notions : l'introduction de drogue au sein de l'entreprise pour son usage personnel ou pour la revendre à d'autres membres du personnel peut être considérée comme une faute grave et même lourde de consé- quences dans le cadre des règles d'hygiène et de sécurité.

Le règlement intérieur doit rappeler l'interdiction de détention et d'usage de substances illicites au sein de l'entreprise et l'obligation de respecter les examens exigés par le médecin du travail, notamment pour les postes à risque.

Il est indispensable d'adopter au sein de l'entreprise une attitude cohérente et une très large concertation au sujet des problèmes de sécurité avec la direction et les délégués du personnel au sein des comités d'hygiène et de sécurité et conditions de travail.

Il faut multiplier les campagnes d'information et créer des cellules de prévention. En cas de résultats positifs, et à condition que la positivité soit confirmée par une autre méthode, le médecin du travail, dans le cadre de la liberté individuelle, peut proposer à l'intéressé d'être dirigé vers un centre de cure. Le médecin de famille est régulièrement confronté à la toxicomanie et aux conduites addictives, il se doit de la prendre en charge. Le médecin du travail a le devoir de s'intéresser à ce phénomène dont les conséquences sont multiples pour la santé et la sécurité de l'individu, de l'entreprise et de la collectivité. Sa mission sera toujours double : protéger le «patient» et protéger l'entreprise en fonction de trois éléments : santé, sécurité et sûreté. 